\title{
Failure Propagation Modeling for Safety Analysis using Causal Bayesian Networks
}

\author{
Mattias Nyberg \\ Royal Institute of Technology (KTH), Stockholm, Sweden, and \\ Scania AB, Södertälje, Sweden \\ mattias.nyberg@scania.com
}

\begin{abstract}
Bayesian Networks (BN) have in previous literature been recognized as a powerful tool for safety analysis, with several advantages over traditional methods such as fault trees. The construction of BNs for safety analysis is however cumbersome; no easier than construction of fault trees. The paper therefore presents a systematic method for construction of BNs for analysis. It is recognized that a special kind of BNs is required, namely Causal BNs. The basic principle to construct these Causal BNs is to utilize specifications of services, or requirements, and their relationships. The approach is especially attractive in the context of safety standards (e.g. ISO26262) where specification and traceability of requirements is already mandatory. The framework in the paper also provides a theoretical link between requirements engineering and the dependability theoretical definitions of fault and failure.
\end{abstract}

\section{Introduction}

Two of the most common, and traditional, methods for safety analysis are fault tree analysis (FTA) and Failure Mode Effects Analysis (FMEA). Recently Bayesian networks has been proposed as an alternative, e.g. see [3, 8]. In its construction, there are many similarities to fault trees, and in fact, any fault tree can be mapped into a Bayesian Network (BN) [3]. BNs have several important advantages compared to fault trees; in particular, the use of probabilistic intermediate nodes instead of only and/or gates, more flexible modeling of dependencies between components, more than two state values of nodes, and the possibility to use powerful computational engines developed for general BN inference.

Even though BNs are powerful, the BN model still needs to be built before safety analysis can be done. The effort may also be greater compared to fault trees, due to the increased modeling power, and thereby a larger amount of modeling choices for the modeler. One option would be to first build a fault tree and then convert it into a BN. However, the first three advantages of using $\mathrm{BNs}$, in the list above, would then be lost. Therefore, to support the engineers, there is a need for a systematic method to construct BNs for safety analysis. A systematic method would also reduce the reliance on personal judgments of the individuals doing the modeling. 
With this motivation, the current paper proposes, as the main contribution, a systematic method for construction of BNs for safety analysis. The aim is a method with a minimal amount of manual steps and human input. The chosen approach is therefore to use as inputs, information already available in a standard engineering development environment: the architecture and the requirements. Both these artifacts are also already mandatory in the context of safety standards, such as ISO 26262.

The development of the proposed method starts in a study of some typical safety analyses tasks. An important finding, and a second contribution of the paper, is that standard BNs are in fact not sufficient. Instead so called Causal BNs [12] must be used. With standard BNs, failure causes cannot be correctly identified, and some types of needed inference computations cannot be performed. This is highlighted by showing that computations of important measures [7] result in wrong answer if standard BNs are used.

The method for constructing the BN is based on building a Failure Propagation (FP) graph, which is then shown to be a BN if complemented with probabilities. The generation of the FP graph is based on an analysis of the structure of the requirements together with the architecture. This is done using a formal framework where dependability theoretical definitions [1] of faults/failure are linked to requirements engineering [6] by viewing components as providers of services. The formalization of this link is a third contribution of the paper. The link is fundamental in order to maintain consistency between the dependability and the requirements engineering view of the system. There are other frameworks that formalize both concepts of failures and requirements, see EAST-ADL [10] and AADL [13]. However, none of these frameworks establish the formal link between the two concepts. Without considering this link, a fault in a component may cause a failure but no requirement is violated, or the opposite, the fault causes a requirement to become violated but no failure occurs. These situations are avoided if the proposed link between requirements and failures is maintained. Using this approach a requirements traceability graph [6] becomes closely related to the FP graph and also a fault tree.

The paper is organized as follows. First, Section 2, introduces a realistic industrial example, used throughout the paper to illustrate the approach. Section 3 then investigates a set of typical safety analyses and their implications on the needed type of BN. Section 4 and 5 present the theoretical framework and the systematic BN construction method respectively. In Section 6, an example scenario is presented and the proposed method is illustrated together with some typical safety analyses. Finally, Section 7 describes some further relations to existing literature.

\section{Illustrative Application Example}

As an introduction to the problem formulation, and as a running example, we will use a simplified version of a Fuel Level Display (FLD) system. This particular example system is found in trucks from the Swedish truck manufacturer Scania 


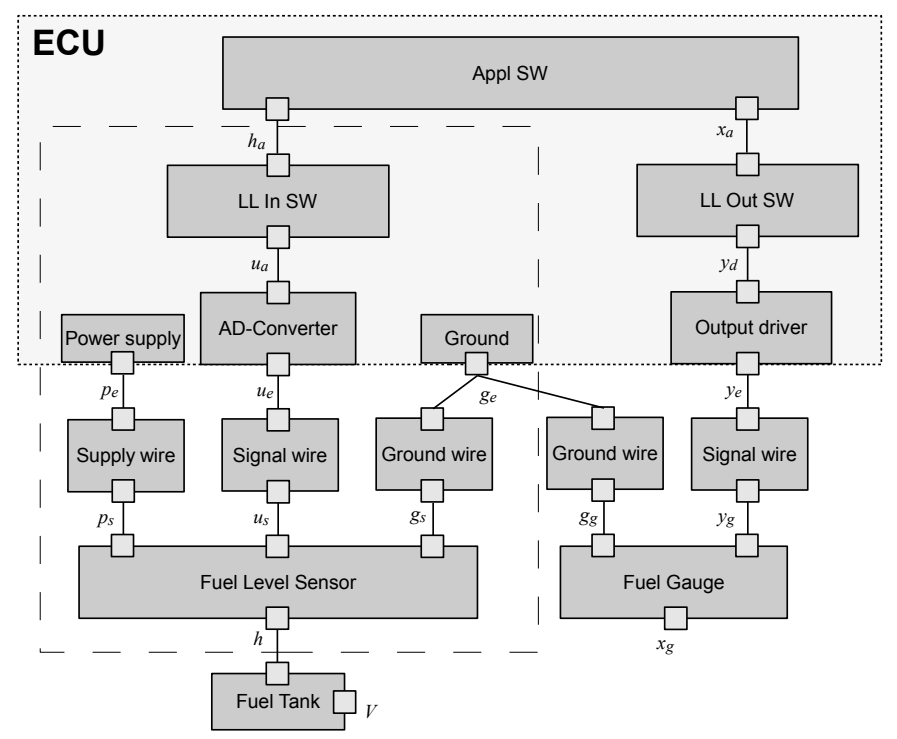

Fig. 1. The FLD (Fuel Level Display) system.

CV AB. The purpose of the system is to, using a gauge, inform the driver about the amount, in percentage, of fuel in the fuel tank. Similar systems are standard equipment in basically all cars and trucks running on liquid fuel. The FLD system is safety critical since run-out-of fuel leads to unexpected loss of engine power with implications of loss of steering, and stops in potentially dangerous locations such as in the middle of a highway.

Fig. 1 shows the architecture of all components and their connections in the system: fuel tank, fuel level sensor, wires connecting the sensor to the ECU (Electronic Control Unit), power supply, ground, AD-converter, low-level input SW converting voltage value to height, application SW converting the height to fuel volume, low-level output SW converting volume to a percentage value, output driver controlling the fuel gauge, wires to the fuel gauge, and the fuel gauge itself. Most of these components are subject to design errors, and all of them are subject to random failures during operation.

Fig. 2 shows a corresponding BN for safety analysis made in the BN-tool Genie [4]. Source nodes, one for each component, represent all possible faults in the system, and non-source nodes represent failures. Links represent propagation of faults to failures, or failures to failures. Safety of the system can be assessed easily by inference computations made by the BN tool, e.g. the overall safety of the system equals the probability of the top node to be in the state "Failed".

Now, the key problem investigated in this paper is: given a system architecture description, like the one in Fig. 1 how to arrive in the BN shown in Fig. 2. 


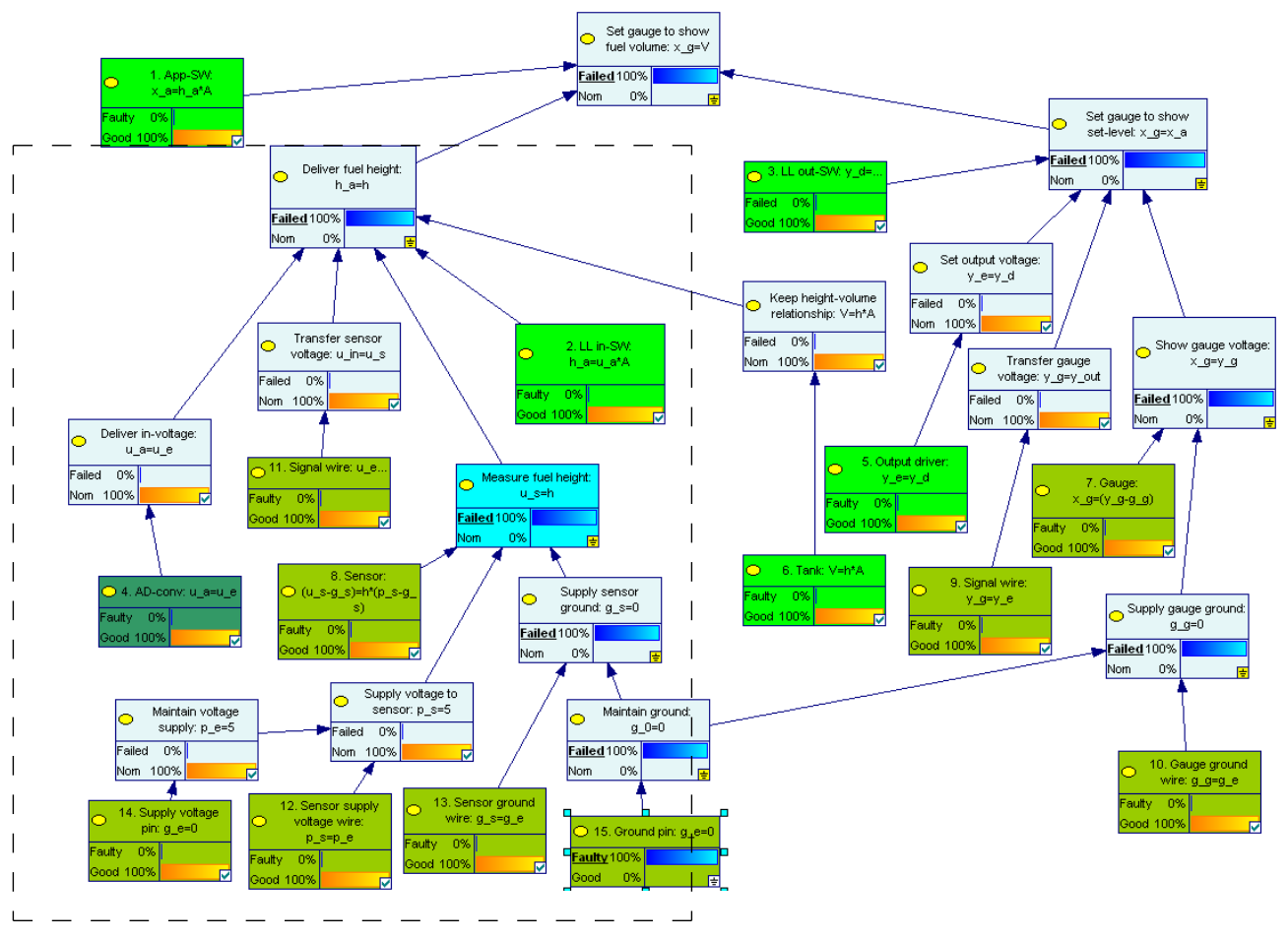

Fig. 2. A Bayesian network modeling failure propagation in the FLD system. 
We are aiming for a systematic method, meaning that two different persons applying the approach should end up in the same BN. The method should also give a BN that is capable of answering queries related to safety analysis, so called safety queries.

\section{Probabilistic Formulation of Safety Analysis}

We will below present a set of typical safety queries and for each of them describe how they can be formulated in a probabilistic framework. We also explain how to obtain an answer to each safety query by using the BN. The typical example of a safety query is "how safe is the system?", but there are also other relevant safety queries as we will see. Note also that more comprehensive analyses like FMEA and FTA can be formulated as a set of more simple queries.

Central in several of the queries is the notion of causality. In accordance with [12], we say that a fault or failure has $F_{c}$ causal influence ${ }^{1}$ on another failure $F_{t}$ if $P\left(F_{t} \mid \operatorname{do}\left(F_{c}\right)\right)>P\left(F_{t}\right)$, where $\operatorname{do}\left(F_{c}\right)$ denotes the intervention that fault $F_{c}$ is injected in the system such that the implied change is local, and does not spread over to mechanisms other than specified by $\operatorname{do}\left(F_{c}\right)$. If $F_{c}$ has a causal influence on $F_{t}$, we also say that $F_{c}$ is a possible cause of $F_{t}$ and that $F_{t}$ is an possible effect of $F_{c}$.

Query I (System Safety) How safe is the system? Safety of a system refers to the absense of a set of identified safety related system failures. The identification of these failures is typically done using hazard identification, e.g. as described in ISO26262. We will use the term top-level failure to refer to these specific failures. In terms of probability, a measure of system safety is $P\left(F_{t}\right)$ where $F_{t}$ is a top-level failure. Provided $F_{t}$ is represented by a node in a BN, the inference $P\left(F_{t}\right)$ is trivial using a BN-tool.

Query II (Failure Effect and Cause) What are the possible top level effects, and what are the possible causes, of a specific component failure? This query is relevant for example in the context of making an FMEA. Provided all considered faults and failures are represented by nodes in a BN, and if the links in the BN represent causal influence, possible causes and effects of a failure $F_{C}$ are trivially found as ancestors and descendents of the node $F_{C}$.

Query III (Component Importance) How important is a specific component with respect to system safety? For evaluation of the importance of a single component for the overall system safety, reliability, availability, etc., a number of importance measures have been proposed in the literature; for an overview see [7]. The basis of these importance measures is a comparison between

\footnotetext{
${ }^{1}$ We asssume that faults can only increase the probability of failure, not decrease.
} 
the unconditioned system reliability and system reliability conditioned on a failure or conditioned on no-failure in the specified component. Two of the most commonly used importance measures are RAW (Reliability Achievement Worth) and RRW (Reliability Reduction Worth). These measures can be expressed as

$$
R R W=\frac{P\left(\neg F_{t}\right)}{P\left(\neg F_{t} \mid F_{C}\right)} \quad R A W=\frac{P\left(\neg F_{t} \mid \neg F_{C}\right)}{P\left(\neg F_{t}\right)}
$$

where $\neg F_{i}$ denotes the absence of failure $F_{i}$. The intention of RRW is to evaluate how much the overall system safety is reduced by a failure in the specified component. The intention of RAW is to evaluate the maximum decrease in system failure probability consequent to an improvement of the specified component.

In RRW, the denominator $P\left(\neg F_{t} \mid F_{C}\right)$ aims at quantifying the absolute reliability resulting from a component failure $F_{C}$. However, the precise meaning of $P\left(\neg F_{t} \mid F_{C}\right)$ is the probability of $\neg F_{t}$ when $F_{C}$ is observed. To capture the intention of the RRW measure, we need instead to use the expression $P\left(\neg F_{t} \mid \operatorname{do}\left(F_{C}\right)\right)$. The issue is highlighted in the following example:

Example 1 Loss of power to the lighting system of a car has the effect that the failure "NoMainLight" becomes present. It also has the effect that the light over the registration sign gets the failure "NoRegistrationLight". The top level failure "NoMainLight" is classified as safety critical. To quantify the importance of the failure "NoRegistrationLight" consider first the expression ${ }^{2}$

$$
P(\text { ("NoMainLight"|do("NoRegistrationLight")) }
$$

Since the intervention to inject the failure "NoRegistrationLight" will not effect the main light, we have the relationship

$P($ "NoMainLight"|do("NoRegistrationLight" $))=P($ "NoMainLight" $)=$ "very low"

By design, the rightmost probability is very low, otherwise the system would not be acceptably safe. Thus, in this particular example, the dominator and numerator in RRW becomes equal, and the RRW measure becomes equal to 1 indicating that the system safety is not reduced by the failure "NoRegistrationLight". This corresponds to a correct quantification of the importance of the failure "NoRegistrationLight".

Now, consider the other alternative

$$
P(\text { "NoMainLight"|"NoRegistrationLight" })=\text { "not so low" }
$$

which according to the discussion above gives the wrong result; the RRW measure becomes larger than 1 .

\footnotetext{
${ }^{2}$ To simplify the evaluation of the probabilities, we consider here the probability of failure instead of probability of no failure as in the RRW measure, e.g. $P($ "NoMainLight"|do("NoRegistrationLight")) instead of $P(\neg " N o M a i n L i g h t " \mid$ do("NoRegistrationLight")).
} 
In conclusion, when component importance is measured, the expression $P\left(\neg F_{t} \mid \operatorname{do}\left(F_{C}\right)\right)$ must be used instead of $P\left(\neg F_{t} \mid F_{C}\right)$. Thus, when using a BN, the ability to compute expressions like $P\left(\neg F_{t} \mid \operatorname{do}\left(F_{C}\right)\right)$ is necessary.

Remark It can be noted that with the use of the expression $P\left(\neg F_{t} \mid \operatorname{do}\left(F_{C}\right)\right)$, the RRW measure becomes a quantification of amount of causal influence, since, in essence, it becomes an evaluation of the difference between $P\left(F_{t} \mid d o\left(F_{c}\right)\right)$ and $P\left(F_{t}\right)$.

\subsection{Requirements on the Bayesian Network}

This section has listed a set of three types of safety queries and formulated them in terms of graph analysis or probabilistic inference in a BN. From this list of queries we can conclude that we need a BN with the following properties: (i) there must be nodes for faults and failures we want to reason about, including top-level failures; (ii) the network must be causal, i.e. links represent causal influence such that possible causes and effects can be identified; (iii) the BN must correspond to a factorization of the joint probability of all considered faults and failures, otherwise inference will in general not give the correct answer.

\section{Framework (for building the Failure Propagation Graph)}

From the previous section, we can conclude that to answer the safety queries, the problem statement of the paper becomes the following:

Given a real world system, with a set of possible faults and failures, derive systematically a Bayesian network fulfilling the requirements in Section 3.1.

This section describes a formal framework with concepts to be used later on when in Section 5 constructing the BN. Because of space limitation, the description of the framework is limited to systems with a flat structure; an extension to hierarchical systems is possible, but comes with more involved notations.

\subsection{Components and Systems}

A component is a set of variables called ports, and is used to model an element of SW or HW. By sharing the same port variable, components can be connected to other components. A component has a non-empty ordered set of behavioral modes, where each represents a relation, i.e. a set of trajecteries, defined on its ports. Note that behavioral modes may be overlapping in the sense that one trajectory may belong to two different behavioral modes. One behavioral mode (the first by convention) is always the no-fault mode (also called good or normal mode). The other behavioral modes model faulty behavior.

A system is a set of, possibly connected, components. 


\subsection{Services}

We also consider relations, on port variables, representing desires from some perspective. In literature, such relations are usually named "requirements", "functions", "services", "capabilities", "promises", or "guarantees". For naming, we will here follow [1] and use the word "service" even though other names may be prefered depending on the tradition in the actual application domain. It is also the experience of the author that in discussions with engineers, the term "service" creates associations corresponding well to the formal definitions presented here.

\subsection{Representation of Behavior and Services}

We assume that both services and behavior are represented by formulas over the ports involved in the relation. For instance, if $y$ and $u$ are two ports, an example of a relation is $y \leq u$. We also consider so called entailment relations between formulas; for example, we write

$$
y=u \mid=y \leq u
$$

to denote that the formula $y=u$ entails the formula $y \leq u$, i.e. all assignments to $y$ and $u$ that satisfy the $y=u$ will also satisfy $y \leq u$. The entailment relation will also be generalized to cases with sets of formulas, on one or both sides of the symbol $\models$.

\subsection{System Design}

In Section 4.1, the concept of system was introduced. We will now introduce a richer structure, called system design, in which services are related to components and to each other. Firstly we allow services to be allocated to components. We will assume that each service is allocated to exactly one component. If service $s$ is allocated to system component $e$, we say that $e$ is a service provider of service $s$.

A service $s_{e}$ allocated to a component $e$ may be associated to one or several services of other components using an association named supplier service. We will say that a component $e_{1}$ is a supplier to a component $e_{2}$ if there is a service of $e_{2}$ that is a supplier service to a service of $e_{2}$.

We consider a system design to be a system together with services allocated to the system components, and where each service is possibly associated to a set of supplier services. That is, a system design is a tuple $\langle S Y S, S, A, U\rangle$, where

- $S Y S$ is a system, i.e. a set of connected components

$-S$ is a set of services, where each service is a relation on the ports in $S Y S$

$-A \subseteq S \times S Y S$ is an allocation of services to components, such that each service is allocated to exactly one component

$-U \subseteq S \times S$ is an association of supplier services to services. 
We say that a design is acyclic if the directed graph formed by the supplier service association is acyclic, i.e. contains no cycles.

Example 2 Consider the part of the FLD system involved in sensing the fuel height. This part is marked with a dashed box in Fig. 1. We assume that there is one service allocated to each component. The nominal behavior and the service $s_{i}$ of each component are as follows:

\begin{tabular}{|c|c|c|}
\hline LL-In SW & $h_{a}=f^{-1}\left(u_{a}\right)$ & $s_{1}: h_{a}=h$ \\
\hline $\mathrm{AD}$ converter & $u_{a}=u_{e}$ & $s_{2}: u_{a}=0.2 u_{e}$ \\
\hline Supply wire & $p_{e}=p_{s}$ & $s_{3}: p_{e}=p_{s}$ \\
\hline Signal wire & $u_{e}=u_{s}$ & $s_{4}: u_{e}=u_{s}$ \\
\hline Ground wire & $g_{e}=g_{s}$ & $s_{5}: g_{e}=g_{s}$ \\
\hline Power supply & $p_{e}=5 \mathrm{~V}$ & $s_{6}: \quad p_{e}=5 \mathrm{~V}$ \\
\hline Ground & $g_{e}=0 V$ & $s_{7}: g_{e}=0 \mathrm{~V}$ \\
\hline Fuel level sensor & $\frac{u_{s}-g_{s}}{p_{s}-g_{s}}=f(h)$ & $s_{8}: \quad u_{s}=5 f(h)$ \\
\hline
\end{tabular}

We assume that the supplier services of the service $s_{1}$, i.e. $h_{a}=h$, are $s_{2}, s_{4}$, and $s_{8}$. Further, we assume that the supplier services of the service $s_{8}$, are $s_{3}$, $s_{5}, s_{6}$, and $s_{7}$.

\subsection{Properties of Services}

Let $B_{N F}(e)$ be an operator returning the no-fault behavior of component $e$. We will now introduce three properties of services needed later on to ensure that the constructed BN does not contain redundant links, i.e. the set of parents of each node is minimal (Markovian [12]).

Definition 1 (Proper Service) Given a design $\mathcal{D}$, a service $s_{e} \in S$, allocated to component $e$, is proper in $\mathcal{D}$ if, for its set of supplier services $s_{1}, \ldots s_{n}$, it holds that

$$
s_{1}, s_{2}, \ldots s_{n}, B_{N F}(e) \models s_{e} .
$$

Definition 2 (Critical Supplier Service / Behavior) Given a design $\mathcal{D}$ with a proper service $s_{e} \in S$ with supplier services $s_{1}, \ldots, s_{n}$, a supplier service $s_{i}$ is critical for $s_{e}$ if

$$
\left\{s_{1}, \ldots s_{n}, B_{N F}(e)\right\} \backslash s_{i} \not \models s_{e}
$$

and the behavior $B_{N F}(e)$ is critical for $s_{e}$ if

$$
\left\{s_{1}, \ldots s_{n}\right\} \not \models s_{e}
$$

Definition 3 (Direct Supplier Services) Given a design $\mathcal{D}$ with a proper service $s_{e}$ allocated to component $e$, let $U$ be the set of supplier services of $s_{e}$ together with the behavior $B_{N F}(e)$. A subset $Q \subseteq U$ is direct if there is no nonempty set $W$ of services, where $s_{e} \notin W$ and $W \cap U=\emptyset$, such that $W \cup(U \backslash Q) \models s_{e}$ and $U \models W$. 


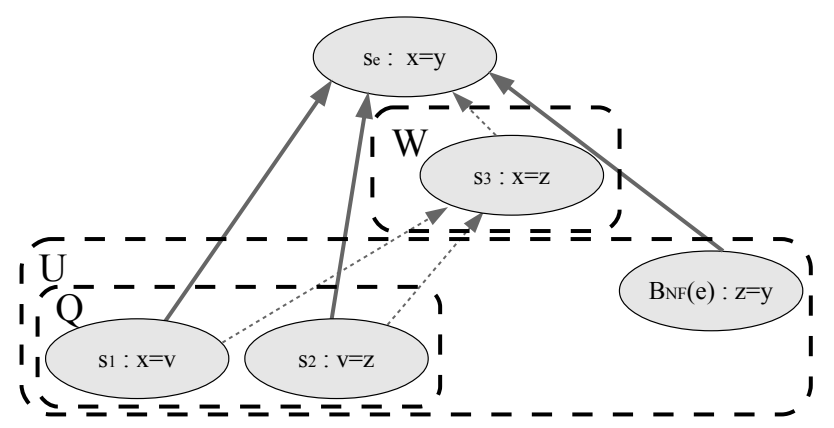

Fig. 3. An example where the subset $Q$ of supplier services is not direct.

Example 3 (Direct supplier services) To illustrate the concept of direct supplier services, consider Fig. 3. Each node represent a service and edges supplier service relations. The solid edges represents a case where the supplier services of $s_{e}$ would not be direct. If instead the two leftmost solid edges would be replaced by the dashed edges, the supplier service $s_{3}$ of $s_{e}$ would be direct.

Example 4 We first investigate if the service $s_{1}$ from Example 2 is proper, i.e. $h_{a}=h$. In fact, properness follows trivially from

$$
h_{a}=f^{-1}\left(u_{a}\right)=f^{-1}\left(u_{e}\right)=f^{-1}\left(u_{s}\right)=f^{-1}(f(h))=h
$$

where we have, from left to right, used the behavior of the LL-In SW, and the services $s_{2}, s_{4}$, and $s_{8}$, to show that the service $h_{a}=h$ is entailed. For this derivation to hold, we need each of the formulas for each of the listed services. Consequently, all supplier services are critical. Lastly, none of the variables in the derivation (9) is contained in any of the other formulas of the services in the system design. Therefore, no set of services used in the derivation can be replaced by any of the other services. This means that all supplier services are direct.

\subsection{Faults and Failures}

In previous sections we have used the notions faults and failure without giving a proper definition. With the framework now in place, we are able to give these notions a formal meaning. Fault and failure have been defined previously in [9] and [1]. The difference is that the definitions given here provides a formal link to services, i.e. requirements. In the definitions, we use a notion of mode assignment that assigns one behavioral mode to each component in a system.

Definition 4 (Internal Fault) Given a mode assignment, a component e has an internal fault if its behavioural mode (in the mode assignment) is not the no-fault mode. 
Definition 5 (Failure) Consider a design $\mathcal{D}=\langle S Y S, S, A, U\rangle$. Given a trajectory of values of the port variables in $S Y S$, consistent with some mode assignment, a component $e \in S Y S$, with service $s_{e}$ allocated to it, has a service $s_{e}$ failure if the formula representing the service $s_{e}$ is not satisfied.

Definition 6 (External Fault) Consider a design $\mathcal{D}=\langle S Y S, S, A, U\rangle$ including a service $s_{1}$, allocated to a component $e_{1}$, with supplier service $s_{2}$ to $s_{1}$, where $s_{2}$ is allocated to component $e_{2}$. Given a trajectory of values of the port variables in $S Y S$, consistent with some mode assignment, the component $e_{1}$ has an external fault with respect to supplier service $s_{2}$ if the component $e_{2}$ has a service $s_{2}$ failure.

\section{Constructing the Bayesian Network}

Given an acyclic design $\mathcal{D}=\langle S Y S, S, A, U\rangle$, let the FP (Failure Propagation) graph be the graph formed by having:

- one node for each service $s_{i} \in S$,

- one node for the behavioral mode $b_{e}$ of each component $e \in S Y S$,

- an arc $\left(b_{e}, s_{e}\right)$ for each service $s_{e}$ allocated component $e$, and

- an $\operatorname{arc}\left(s_{i}, s_{e}\right)$ if and only if $s_{i}$ is a supplier service of $s_{e}$.

Corresponding to the nodes in the FP graph, we view all $s_{i}$ and $b_{e}$ as discrete random variables. Each service variable $s_{i}$ takes the value "Nom", if the formula representing the service is satisfied, and "Failed" otherwise. We make the following two assumptions, in which $p a^{G}\left(s_{i}\right)$ means the parents of the node $s_{i}$ in the FP graph.

Assumption 1 If a service $s_{i}$ is proper and its set of supplier services direct, then the local Markov property with respect to the FP graph holds, i.e. it holds that $P\left(s_{i} \mid p a\left(s_{i}\right)\right)=P\left(s_{i} \mid p a\left(s_{i}\right), X\right)$ for all sets of non-descendents $X$.

Assumption 2 It is possible to manipulate the corresponding real world system such that a service $s_{i}$ becomes not satisfied, and also such that a certain behavioral mode of a component can be enforced, and in both cases, if Assumption 1 holds, the local Markov property continues to hold for all other nodes, w.r.t. the FP graph, also in the manipulated system.

A manipulation fulfilling Assumption 2 is denoted $\operatorname{do}\left(s_{i}=\right.$ Failed $)$, or $\operatorname{do}\left(b_{e}=\right.$ $m$ ) for the behavioral mode $m$.

We will now introduce the concept of causal Bayesian network from [12]:

Definition 7 (Causal Bayesian Network) A directected acyclic graph $G$ is $a$ causal Bayesian network for a set of random variables $V$ if for each $X \subseteq V$, it holds that

$$
P(v \mid d o(X=x))=\prod_{i, V_{i} \notin X} P\left(v_{i} \mid p a^{G}\left(v_{i}\right)\right)
$$

whenever $v$ is consistent with $X=x$. 
The key property of a causal Bayesian network is that links represent causal influence $^{3}$ and therefore any probability $P(v \mid w, \operatorname{do}(X=x))$ can be computed easily by so called interventional calculus, i.e. incoming links to $X$ are deleted and we then compute $P(v \mid w, x)$. Interventional calculus is also commonly supported in BN tools explicitly, e.g. in Genie.

The main result of the paper is now presented as a theorem. The proof utlilizes Assumption 1 and 2 but is omitted because of space limitations. It can be found in Appendix.

Theorem 1 Consider an acyclic design $\mathcal{D}$, where for each service $s_{i}$ it holds that

- it is proper,

- its set of supplier services is direct, and

- each of its supplier services and its behavior is critical.

The FP graph of $\mathcal{D}$, is a causal Bayesian network for the set of discrete variables, one for each of the services $S$ and one for each of the components in SYS. Furthermore, the set of parents of each node in the FP graph is minimal (Markovian).

Example 5 To construct a causal BN for the sensor part of the FLD-system from Example 2, we use Theorem 1. In Example 4, we already concluded that all services are proper with direct and critical supplier services. Considering applicability of Theorem 1, we show that Assumption 2 holds.

The FP graph obtained from stated service relations is in Fig. 2 seen as the part inside the dashed box. The node for service $s_{1}$ (named "Deliver fuel height") has 5 parents. We need to find a manipulation of the real world system such that the service $s_{1}$ becomes not fulfilled, i.e. $h_{a} \neq h$ while ensuring that the local Markov property holds for all other nodes. One such manipulation is to inject a fault in the signal between the LL-In SW and the Appl SW.

\subsection{Conditional Probability Tables (CPT)}

The actual probability values in the CPT associated to each node, is given partly by the framework of the paper. Firstly, for the case all parents are in the "nofault" /"Nom" state, the probability of failure is 0 due to the relation (6). Secondly, in cases where not fulfillment of the no-fault behavior of a component, or not fulfillment of a supplier service, logically implies not fulfillment of a service, the probability of failure is 1 .

The rest of the CPT probabilities need to be obtained from expert knowledge, experiments, or using standard default values. For example, values of fault probabilities in source nodes can be obtained from component suppliers or standards. Regardless of how the values determined, important for most safety analyses is that every value is not underestimated. That is, if there is any amount of uncertainty of an exact value, the user must find a value that is an upper bound,

\footnotetext{
$\overline{{ }^{3} \text { See Appendix. }}$
} 
guaranteeing that the actual probability value is below that bound. Following this rule, it is guaranteed that the safety query I from Section 3, results in an upper bound of the actual failure probability.

\section{Example Scenario}

For an illustration of the use of the approach, we here extend the discussion about the FLD system. One service was defined and allocated to each of the components. The approach described and summarized in Theorem 1 was then used to construct the BN shown in Fig 2. Probability values in the CPTs were determined using the principles of Section 5.1. So called Noisy-Or nodes [4] were utilized to reduce the number of probabilities to enter. Because of the importance to not underestimate failure probabilities, most probabilities of failure, given a failure of any of the supplier services, were in fact set to one.

Safety query of type I, computed by providing no evidence to the inference engine, resulted in that the safety, i.e. the top level failure $P\left({ }^{\prime} x_{g}=V^{\prime}=\right.$ Failed $)=9.4 e-8$. This level of failure probability was considered to be too high, so therefore, the important measure RAW, i.e. safety queries of type III, was utilized to identify the components contributing most to the non-safety of the system. For example, considering the numerator in the RAW, see (1), we have $P\left({ }^{\prime} x_{g}=V^{\prime}=\right.$ Failed $\left.\right|^{\prime} g_{e}=0^{\prime}=$ Good $)=1 e-8$; the denominator is less relevant since it is constant in this comparative analysis. These queries gave the result that the components contributing mostly to the non-safety were the fuel level sensor, the fuel gauge, the 5 wires, the power supply, and ground.

To improve the safety level of the system, it was determined that the system should be redesigned: a new gauge with increased robustness and with a safe state, an addition of a safety mechanism in the Low-Level-In SW detecting electrical failures, a mechanism for fault accommodation utilizing the gauge safe state based on input from the fault detection. This resulted in a new fault mode of the gauge denoted "Off", and three new services related to the fault detection and accommodation. Theorem 1 was used to construct the corresponding new $\mathrm{BN}$. Using this new $\mathrm{BN}$, the total safety was estimated to be $P\left({ }^{\prime} x_{g}=V^{\prime}=\right.$ Failed $)=9 e-9$, an improvement of a factor 10 .

\section{Related Work}

So called Assume-Guarantee contracts have been proposed as a mean to structure requirements around components [2]. The concepts of services proposed in this paper is in fact highly compatible with Assume-Guarantee contracts. When composing a set of components with contracts into a system, the guarantees can be viewed as services. If an assumption of one component is satisfied by guarantees of other components, these guarantees become supplier services, and these other components become suppliers. If instead, the here proposed framework of services is used as the basis for structuring the requirements, a service 
of a component can be interpreted as a guarantee, and its supplier services as assumptions.

The literature contains many other approaches to failure propagation modeling, for an overview see [5]. In these approaches, failures and their propagation are explicitly stated in the model, such as in EAST-ADL [10] and AADL [13]. This stands in contrast to the approach proposed in the current paper, where fault propagation is derived implicilty from services, i.e. requirements. If desired, the obtained FP graph can then be translated into e.g. annotations in EAST-ADL or AADL. The advantage with the here proposed approach is that consistency between requirements and failures is enforced.

\section{Conclusions}

In the paper, we have first investigated what type of BN that is needed when the target is safety analysis. The conclusion, and a first contribution, is that Causal BNs are needed. In fact, if standard BNs are used, incorrect results may be obtained, such in the examples of the important measures RAW and RRW. In the framework set up in Section 4, a formal link has been made between services, i.e. requirements, and the dependability theoretical $[1,9]$ concepts of fault and failure; a second contribution of the paper. The main contribution is the constructive method for building causal BNs for safety analysis of real world systems. The method takes as input, an architectural description, such the one in Fig. 1, and the set of requirements, and produces as output a causal BN, such as the one in Fig. 2.

\section{References}

1. Avizienis: Toward systematic design of fault-tolerant sys. Computer 30(4), 51-58 (1997)

2. Benveniste, A., et al.: Multiple viewpoint contract-based specification and design. In: Formal Methods for Components and Objects. pp. 200-225. Springer (2008)

3. Bobbio, A., Portinale, L., Minichino, M., Ciancamerla, E.: Improving the analysis of dependable systems by mapping fault trees into bayesian networks. Reliability Engineering \& System Safety 71(3), 249-260 (2001)

4. GeNIe: (2010), university of Pittsburgh, URL: http://genie.sis.pitt.edu/

5. Grunske, Han: A comparative study into architecture-based safety evaluation methodologies using aadl's error annex and failure propagation models. In: High Assurance Systems Engineering Symposium, 2008. HASE 2008. 11th IEEE. pp. 283-292. IEEE (2008)

6. Hull, E., Jackson, K., Dick, J.: Requirements Engineering. Springer London (2011)

7. Jose F. Espiritu, a.W.C., Prakash, U.: Component criticality importance measures for the power industry. Electric Power Systems Research (77), 407 - 420 (2007)

8. Langseth, H., Portinale, L.: Bayesian networks in reliability. Reliability Engineering \& System Safety 92(1), 92-108 (2007)

9. Laprie, J. (ed.): Dependability: Basic Concepts and Terminology. Springer (1991) 
10. MAENAD: EAST-ADL domain model specification. Tech. rep., URL:www.maenad.eu/public/EAST-ADL-Specification_M2.1.10.20120629.pdf (2012)

11. Nyberg, M.: Failure propagation modeling for safety analysis using causal Bayesian networks. Tech. rep., KTH (2013)

12. Pearl, J.: Causality: Models, Reasoning and Inference. Cambridge Uni. Press, 2 edn. (2009)

13. P.H.Feiler, A.-E.Rugina: Dependability modeling with the Architecture Analysis and Design Language (AADL). Tech. rep., CMU/SEI-2007-TN-043 (2007) 


\section{Appendix}

\section{A.1 Bayesian Networks}

To distinguish between different variants of definition of Bayesian network existing in the literature, we introduce two definitions of Bayesian network.

Definition 8 (Markovian Bayesian Network [12]) A DAG G is a Markovian Bayesian network for a set of variables $V$, if the set of nodes in $G$ represents the set $V$, and the set of parents of $V_{i}$ in $G$ are Markovian parents of $V_{i}$.

Definition 9 (Bayesian Network [12]) A DAG G is a Bayesian network for a set of variables $V$, if the set of nodes in $G$ represents the set $V$, and the set of parents of $V_{i}$ in $G$ are a super-set of a set of Markovian parents of $V_{i}$, or simply stated, $P(v)$ admits a factorization according to $G$.

Proposition 1 (Bayesian Network vs Markovian Bayesian Network [12]) A DAG $G$ is a Bayesian network for $V$ if $G$ is a Markovian Bayesian Network for $V$.

Theorem 2 (Parental Markov Condition [12]) A DAG G, where the set of nodes in $G$ represents a set of variables $V$, is a Bayesian network for $V$ if and only if each variable $V_{i}$ is independent of all its nondescendants in $G$, conditional on its parents in $G$, i.e.

$$
P\left(v_{i} \mid p a_{i}^{G}\right)=P\left(v_{i} \mid p a_{i}^{G}, x\right)
$$

for any set $X$ of nondescendants of $V_{i}$.

\section{A.2 Causality}

Assume that for each variable $X$ and value $x$, there is an associated manipulation of the real system setting $X=x$, denoted $\operatorname{do}(X=x)$.

Definition 10 (Causal Influence [12]) A variable $V_{i}$ has a causal influence on $V_{j}$ if the distribution of $V_{j}$ is sensitive to actions $d o\left(V_{i}=v_{i}\right)$, i.e. if there exist a value $v_{i}$ such that

$$
P\left(v_{j} \mid d o\left(V_{i}=v_{i}\right)\right) \neq P\left(v_{j}\right)
$$

Definition 7 (Causal Bayesian Network [12]) A DAG G is a causal Bayesian network for $V$ if for each $X \subseteq V$, it holds that $P(v \mid d o(X=x))$ admits a factorization according to

$$
P(v \mid d o(X=x))=\prod_{i, V_{i} \notin X} P\left(v_{i} \mid p a_{i}^{G}\right)
$$

whenever $v$ is consistent with $X=x$, where $p a_{i}^{G}$ refers to parents in $G$. 
Note that a Causal $\mathrm{BN}$ is also a $\mathrm{BN}$, since a special case is when $X=\emptyset$, and (10) then becomes equivalent to the property that $P(v)$ admits a factorization according to $G$.

Theorem 3 (Causal BN vs Causal Influence) Let a DAG G be a causal $B N$ for a set of variables $V$, and let $V_{i}$ and $V_{j}$ be any two variables in $V$. Then, if $V_{i}$ has a causal influence on $V_{j}$, then there is a path in $G$ from $V_{i}$ to $V_{j}$.

Proof. Assume $V_{i}$ has a causal influence on $V_{j}$. This means that $P\left(v_{j} \mid \operatorname{do}\left(V_{i}=\right.\right.$ $\left.\left.v_{i}\right)\right) \neq P\left(v_{j}\right)$. Assume contradictory that there is no path in $G$ from $V_{i}$ to $V_{j}$. Since there is no path from $V_{i}$ to $V_{j}, V_{i}$ is not an ancestor of $V_{j}$. The intervention $\operatorname{do}\left(V_{i}=v_{i}\right)$ only cuts off incoming links to $V_{i}$ so the incoming links to all ancestors of $V_{j}$ are intact. If we let $V_{j+1}, \ldots V_{N}$ denote the ancestors of $V_{j}$, this means that for the probability distribution of the variables $V_{j}, V_{j+1}, \ldots V_{N}$ under $\operatorname{do}\left(V_{i}=v_{i}\right)$ it holds

$$
P\left(v_{j}, v_{j+1}, \ldots v_{N} \mid \operatorname{do}\left(V_{i}=v_{i}\right)\right)=\prod_{k=j}^{N} P\left(v_{k} \mid p a_{k}^{G}\right)=P\left(v_{j}, v_{j+1}, \ldots v_{N}\right)
$$

We can then compute $P\left(v_{j} \mid \operatorname{do}\left(V_{i}=v_{i}\right)\right)$ as

$$
\begin{aligned}
P\left(v_{j} \mid \operatorname{do}\left(V_{i}=v_{i}\right)\right)=\sum_{v_{j+1}, \ldots v_{N}} P\left(v_{j}, v_{j+1}, \ldots v_{N} \mid \operatorname{do}\left(V_{i}=v_{i}\right)\right)= & \\
= & \sum_{v_{j+1}, \ldots v_{N}} P\left(v_{j}, v_{j+1}, \ldots v_{N}\right)=P\left(v_{j}\right)
\end{aligned}
$$

This contradicts the given fact that $V_{i}$ has a causal influence on $V_{j}$, and therefore, it must hold that there is a path in $G$ from $V_{i}$ to $V_{j}$.

Note that we can have a causal BN in which there is an $\operatorname{arc}$ from $V_{i}$ to $V_{j}$, but $V_{i}$ has not a causal influence on $V_{j}$. That is, not all arcs in $G$ represents causal influence. On the other hand, always, when a node $V_{i}$ has a causal influence on $V_{j}$, there will be a corresponding arc in $G$.

Example 6 Consider a causal $B N$ for a set of binary variables $\left\{V_{1}, V_{2}\right\}$ and with only one arc (i.e. a path), from $V_{2}$ to $V_{1}$. The CPT for $V_{1}$ is defined by $P\left(v_{1}=0 \mid V_{2}=0\right)=P\left(v_{1}=0 \mid V_{2}=1\right)=0.3$. This is a $B N$ for $V$ but $V_{1}$ does not have Markovian parents. By definition of causal BN, we have

$$
\begin{aligned}
P\left(v_{1}, v_{2}\right) & =P\left(v_{1} \mid v_{2}\right) P\left(v_{2}\right) \\
P\left(v_{1}, v_{2} \mid d o\left(V_{1}=v_{1}\right)\right) & =P\left(v_{2}\right) \\
P\left(v_{1}, v_{2} \mid d o\left(V_{2}=v_{2}\right)\right) & =P\left(v_{1} \mid v_{2}\right) \\
P\left(v_{1}, v_{2} \mid d o\left(V_{1}=v_{1}, V_{2}=v_{2}\right)\right) & =1
\end{aligned}
$$

and also

$$
P\left(v_{1} \mid d o\left(V_{2}=v_{2}\right)\right)=P\left(v_{1} \mid v_{2}\right)=P\left(v_{1}\right)
$$


where the last equality holds for the particular considered CPT. This means that $V_{2}$ has not a causal influence on $V_{1}$.

If the BN is Markovian, then all arcs represent causal influence. However, we could still have longer paths that do not represent causal influence, i.e. the relation causal influence is not transitive. This is illustrated in the following example.

Example 7 Consider a DAG $G$ with nodes representing the binary variables $\{A, B, C, D\}$ and arcs $D \rightarrow B, D \rightarrow C, B \rightarrow A$, and $C \rightarrow A$. Assume

$$
\begin{aligned}
P(A=1 \mid B, C) & =[0.4,0.1,0.1,0.4]) \\
P(B=1 \mid D) & =[0.8,0.2] \\
P(C=1 \mid D) & =[0.2,0.8] \\
P(D=1) & =0.7
\end{aligned}
$$

Note that for each variable, the set of parents in $G$ is Markovian. Thus the DAG $G$ is both a $B N$ and a Markovian $B N$. It can be realized that $P(A \mid D)=P(A)$. This means that if $G$ is a causal $B N$, it also holds that $P(A \mid \operatorname{do}(D))=P(A)$. That is, the variable $D$ does not have a causal influence on the variable $A$, but still, there is a path from $D$ to $A$ in $G$.

\section{A.3 FP Graph vs Causal BN}

Theorem 1 Consider an acyclic design $\mathcal{D}$, where for each service $s_{i}$ it holds that

- it is proper,

- its set of supplier services is direct, and

- each of its supplier services and its behavior is critical.

The FP graph of $\mathcal{D}$, is a causal BN for the set of discrete variables, one for each of the services $S$ and one for each of the components in SYS. Furthermore, the set of parents of each node in the FP graph is minimal (Markovian).

Proof. Firstly, from the given fact that each service is proper and direct, together with Assumption 1, it follows that the local Markov property, with respect to the FP graph, holds for each considered discrete variable. This fact holds for each variable in the case of no intervention. In the case of intervention, we need also to use Assumption 2 to conclude that the fact holds for the variables not included in the intervention. Then, Theorem 2 implies that the FP graph, but with incoming arcs to the nodes included in the intervention removed, is a $\mathrm{BN}$ for the considered set of discrete variables. According to the definition of BN, i.e. Definition 9 , the distribution $P(V \mid \operatorname{do}(X=x))$ factorizes according to (13).

To improve readability, we include also a more detailed explanation, and without a use of Theorem 2. In general we have

$$
P(V \mid \operatorname{do}(X=x))=\prod_{i} P\left(v_{i} \mid v_{i+1}, \ldots, v_{n}, \operatorname{do}(X=x)\right)
$$


where the variable ordering is such that all ancestors of $V_{i}$ are among $V_{i+1}, \ldots, V_{n}$. We consider the case when $v_{i}$ is consistent with $X$. If $V_{i} \in X$, then

$$
P\left(v_{i} \mid v_{i+1}, \ldots, v_{n}, \operatorname{do}(X=x)\right)=1
$$

For the case $V_{i} \notin X$, the local Markov property holds according to Assumption 2. This means that

$$
P\left(v_{i} \mid v_{i+1}, \ldots, v_{n}, \operatorname{do}(X=x)\right)=P\left(v_{i} \mid p a_{i}^{G}\right)
$$

Consequently, we have

$$
P(V \mid \operatorname{do}(X=x))=\prod_{i} P\left(v_{i} \mid v_{i+1}, \ldots, v_{n}, \operatorname{do}(X=x)\right)=\prod_{i, V_{i} \notin X} P\left(v_{i} \mid p a_{i}^{G}\right)
$$

Thus, we have shown that the distribution $P(V \mid \operatorname{do}(X=x))$ factorizes according to (13).

Finally, we will prove that each node has Markovian parents. Firstly, note that since $s_{i}$ is proper it holds that $s_{1}, \ldots, s_{n}, b_{i}=s_{i}$, where the $s_{1}, \ldots, s_{n}, b_{i}$ are the parents in the FP graph. This is equivalent to that $P\left(s_{i}=N o m \mid P A_{i}^{G}=\right.$ $[$ Nom,$\ldots$, Nom, NoFault $])=1$. Now assume that some node has not Markovian parents. That is, there is a proper subset $Z \subset P A_{i}^{G}$ such that $P\left(s_{i} \mid p a_{i}^{G}\right)=$ $P\left(s_{i} \mid z\right)$. This would imply $P\left(s_{i}=N o m \mid Z=[N o m, \ldots, N o F\right.$ ault $\left.]\right)=1$ which is equivalent to that $Z=[N o m, \ldots, N o F$ ault $] \models s_{i}$. This contradicts the fact that each service and the behavior in $\left\{s_{1}, \ldots, s_{n}, b_{i}\right\}$ is critical. Thus, by contradiction, we have proven that the set of parents of each node is Markovian. 